\title{
LA CLÁUSULA SOCIAL EN EL CONTEXTO HISTÓRICO DE LAS RELACIONES COMERCIALES "UNIÓN EUROPEA-AMÉRICA LATINA”
}

\author{
Armando Alvares GARCIA JÚNIOR*
}

\begin{abstract}
* Profesor de derecho internacional público, derecho del comercio internacional y sistemas jurídicos no occidentales (grado en derecho), estructura económica mundial, negocios internacionales, comercio exterior y dirección estratégica y política de empresa - I (grado en ADE) y negociación y marketing internacional (MBA-ADE) en la Universidad Internacional de la Rioja, España. Ocasionalmente profesor de dirección estratégica y política de la empresa - II (curso de adaptación en ADE) por la misma universidad. Miembro del Grupo de Investigación "Culturas, religiones y derechos humanos en la sociedad actual" de la Universidad Internacional de la Rioja (GDI-I). armando.alvares@unir.net
\end{abstract}

RESUMEN: El artículo analiza la cláusula social en el contexto histórico de las relaciones comerciales "Unión Europea-América Latina".

PALABRAS CLAVE: cláusula social, derechos humanos, Unión Europea, América Latina, relaciones comerciales.

\section{SOCIAL CLAUSE IN THE HISTORICAL CONTEXT OF TRADE RELATIONS "EUROPEAN UNION-LATIN AMERICA"}

\begin{abstract}
The article analyzes the social clause in the historical context of trade relations "European Union-Latin America".
\end{abstract}

KEYWORDS: social clause, human rights, European Union, Latin America, trade relations.

Recebido em: 16/10/2015 - Aprovado em: 15/11/2015 - Disponibilizado em: 18/12//2015

\section{INTRODUCCIÓN}

Durante el año 2015, el revuelo generado por ciertos agentes políticos, económicos y sociales en Europa, en virtud de las actuales negociaciones del Transatlantic Trade and Investment Partnership (TTIP), negociado entre los Estados Unidos y la Unión Europea, ha sido similar al que ocurre en otras partes del planeta siempre que se discute el binomio "libre comercio-derechos humanos". Con efecto, diversas voces volvieron a clamar por el fin de las violaciones de los derechos humanos y laborales perpetradas por las grandes empresas multinacionales (que, en el caso de ciertos países en desarrollo, son beneficiadas por cierta indiferencia o hasta mismo connivencia de gobiernos corruptos o deseosos de que las exportaciones, a cualquier precio, ayuden a equilibrar las balanzas comercial y de pagos). No se puede ignorar que, en varios países (especialmente en los más pobres), la producción de bienes se realiza en un entorno 
de precarización de las condiciones humanas y laborales, cuya primera víctima, el trabajador, muchas veces evita manifestarse en contra de sus empleadores por el temor de perder la pequeña fuente de ingresos económicos que permita subsistir su familia. Lamentablemente, en Asia, en África y en América Latina, las condiciones laborales, en la práctica, aun están muy lejos del ideal para una enorme parcela de la población, con destaque para los trabajadores poco calificados. En los países centroamericanos, por ejemplo, sigue existiendo una elevada tasa de utilización de trabajo infantil (incluso defendido por las familias más pobres), así como el sistemático incumplimiento de diversas normas de protección del trabajador, como las que estipulan límites de tiempo para las jornadas laborales.

Como se puede suponer, existen muchas dificultades políticas, económicas y operacionales, especialmente en países con gobiernos corruptos e instituciones débiles, para que se establezca un riguroso control sobre las empresas y su conducta (ni siempre adecuada o legal) hacia los trabajadores. Una de las dificultades, cuestionable bajo la perspectiva jurídica, se asocia al hecho de que los trabajadores cuyos derechos son violados no integran las plantillas de las grandes empresas, en virtud del elevado número de contratas y subcontratas en la cadena de producción. Además, como señala de la Garza $(2012)^{1}$, ni todas las empresas pueden, o desean, tener control sobre las condiciones laborales de estas personas, trabajadoras de sus diversos proveedores. No obstante, cuando alguna tragedia humana de grandes proporciones traspasa a los medios de comunicación de masa y salpica a una de esas grandes empresas, se observa un rápido movimiento empresarial que se refleja en declaraciones de ejecutivos publicadas en la prensa $\mathrm{y}$, jurídicamente, en la voluntaria incorporación de Códigos de Buenas Prácticas o reglas de buena conducta, casi siempre de naturaleza sectorial (Bahillo, 2012) ${ }^{2}$.

\section{DERECHOS HUMANOS Y EMPRESA}

En un mercado caracterizado por la elevada competencia, la presión ejercida sobre los trabajadores suele ser vista frecuentemente como una necesidad empresarial teóricamente comprensible: el abaratamiento de costes (mano de obra barata significa, casi siempre, producción con precios competitivos). Los bienes producidos de esta manera (v.g., en las fábricas chinas y bangladesíes) son, en su mayor parte, exportados a mercados

\footnotetext{
1 de la Garza, E. (2012). La subcontratación y la acumulación de capital en el nivel global. pp. 17-40, en Celis J., (2012) Comp. La Subcontratación Laboral en América Latina: Miradas Multidimensionales. Medellín, Colombia: Escuela Nacional Sindical.

2 Bahillo, C. G. (2012). Ética y responsabilidad empresarial en la sociedad globalizada. Revista Icade. Publicación de las Facultades de Derecho y Ciencias Económicas y Empresariales 75, 125-142.
} 
consumidores con mayor poder adquisitivo (Chan, 1998) ${ }^{3}$. Evidentemente, el mundo empresarial no se limita al empleo de mano de obra barata (con o sin violación de los derechos humanos y laborales), puesto que deben ser considerados también muchos otros elementos como, por ejemplo, el acceso a las materiasprimas, la apertura y disponibilidad de lineas de financiación, los acuerdos clave con proveedores o clientes, las ayudas gubernamentales etc. Con efecto, existen diversas matrices de análisis en el campo de la Administración y Dirección de Empresas que posibilitan una perspectiva más cercana a la realidad de la empresa y a la realidad externa (PESTEL, DAFO etc.), además de las ya clásicas "cinco fuerzas de Porter" (Porter, 2008) ${ }^{4}$ que engloban: 1) el poder de negociación de los clientes, 2) el poder de negociación de los proveedores, 3) la amenaza de la entrada de nuevos competidores (barreras de entrada), 4) la amenaza de productos sustitutivos y 5) la relación entre las empresas competidoras. Todos estos factores, y muchos otros, confluyen en un escenario conformado por relaciones de diversa índole ${ }^{5} \mathrm{y}$

\footnotetext{
${ }^{3}$ Chan, A. (1998). Labor standards and human rights: The case of Chinese workers under market socialism. Human Rights Quarterly, 20(4), 886-904.

${ }^{4}$ Porter, M. (2008). Las cinco fuerzas competitivas que le dan forma a la estrategia. Harvard Business Review, 86(1), 58-77.

${ }^{5}$ Las de naturaleza comercial constituyen solamente una de ellas, aunque tremendamente importante para las empresas y para los países o grupos de países (como la Unión Europea o el Mercosur).
}

caracterizado por la intensa competencia en el ámbito internacional. De hecho, el mundo contemporáneo está constituido por extensas redes de poder, como afirma Rezende Martins $(2002)^{6}$, en que la política, la economía, las finanzas, el comercio, la cultura etc. se entremezclan en escala global. Este es el escenario transversal - marcado por la libre competencia y el liberalismo económico - en que se desarrolla la denominada cláusula social, incorporada con el estatus de "cláusula fundamental" (con base en la Convención de Viena sobre el Derecho de los Tratados) en los acuerdos bilaterales de comercio celebrados entre la Unión Europea y los países en desarrollo. Estos distintos factores, estrechamente interconectados (política, economía, finanzas, comercio etc.), hoy parecen algo bastante natural gracias al enorme esfuerzo de historiadores de la talla de Renouvin y Braudel, figuras clave en la historia de las relaciones internacionales en el siglo XX. Sin embargo, muchos obstáculos impuestos por las elites intelectuales $\mathrm{y}$ académicas de Francia, y especialmente de Alemania, han tenido que ser superadas para que la interpretación histórica, antes basada en los grandes eventos militares y biografías de personajes ilustres, pudiera ser aceptada $\mathrm{y}$ comprendida como un crisol de elementos interrelacionados que se mueven paralelamente

\footnotetext{
6 de Rezende Martins, E. C. (2002). Relações internacionais: cultura e poder. Ibri.
} 
en diferentes estructuras temporales (larga, media y corta duración, según Braudel), en un proceso que, evidentemente, no puede y no debe ser confundido con la decisión "momentánea" adoptada por los dirigentes políticos del estado.

\section{LA INCLUSIÓN DE LOS DERECHOS HUMANOS EN EL COMERCIO UNIÓN EUROPEA-PAÍSES EN DESARROLLO}

En este amplio y complejo contexto, la inserción del tema de la protección de los derechos humanos y laborales en las relaciones comerciales Unión Europea-América Latina, mediante la inclusión de la cláusula social en sus acuerdos bilaterales, ocupa un rol que ha logrado cristalizarse en el tiempo, aunque su efectividad, por las propias características de un mercado liberal (donde el agente privado decide sobre la asignación de sus recursos económicos y financieros, lo que incluye pagar bajos salarios), sea verdaderamente cuestionable (Smith, 1998) ${ }^{7}$. De hecho, no existe ninguna prueba de que la cláusula social pueda garantizar el respeto de la dignidad humana y la protección de los derechos humanos y libertades fundamentales en ningún país aunque, como señalaría Ishay $(2008)^{8}$, se

\footnotetext{
${ }^{7}$ Smith, K. E. (1998). The use of political conditionality in the EU's relations with third countries: How effective? European Foreign Affairs Review, 3(2), 253274.

${ }^{8}$ Ishay, M. R. (2008). The history of human rights: From ancient times to the globalization era. Univ of California Press.
}

trate de una iniciativa "políticamente correcta" para su promoción en todos los países en desarrollo. En la realidad, como observan Dunne \& Wheeler $(1999)^{9}$, la protección de esos derechos se contempla cada vez más en las agendas bilaterales, regionales $\mathrm{y}$ multilaterales y en las constituciones políticas de los países ${ }^{10}$.

En lo que concierne a la Unión Europea, por razones históricas, el respeto por los derechos humanos lograron incorporarse como valor fundamental (al lado de la democracia y el estado de derecho) ${ }^{11}$ en el seno de sus normativas fundamentales, incluyendo aquellas que orientan su política exterior y comercial, de modo que la escrupulosa observancia de la cláusula social (y, por extensión, de todos los instrumentos internacionales de protección de los derechos humanos y laborales a ella asociados) se ha convertido en una condición esencial para que los países en desarrollo puedan obtener rebajas arancelarias en sus exportaciones hacia el mercado único. Esto significa que además del rol de promotora de los derechos humanos y laborales la cláusula

\footnotetext{
${ }^{9}$ Dunne, T., \& Wheeler, N. J. (1999). Human rights in global politics. Cambridge: Cambridge University Press. ${ }^{10} \mathrm{De}$ hecho, la política externa actual de los estados occidentales tiende a incorporar y a exaltar los derechos humanos (como demuestra, por ejemplo, el art. 4, II de la Constitución brasileña: "La República Federativa de Brasil se rige en sus relaciones internacionales por los siguientes principios: prevalencia de los derechos humanos".

${ }^{11}$ Por ejemplo, en el Tratado de la Unión Europea de 7 de febrero de 1992 (art. 21).
} 
social también representa una condicionalidad de acceso al mercado interior en condiciones comercialmente ventajosas. Particularmente, la Comisión Europea y el Parlamento Europeo pasaron a adoptar una postura más crítica en relación a los países en desarrollo beneficiados por las rebajas arancelarias unilaterales contempladas en el ámbito del Sistema de Preferencias Generalizadas ${ }^{12}$ en lo que se refiere a su conducta hacia la protección de los derechos humanos y laborales. Por su vez, los sindicatos europeos (con destaque para los franceses) también eran contrarios a las rebajas de aranceles (tanto en los acuerdos recíprocos como en los no recíprocos) y, sobretodo, defendían fervorosamente la inclusión de la cláusula social en el sistema multilateral de comercio $^{13}$ aunque no por altruismo, sino por miedo a perder puestos de trabajo, ya que los precios de los productos obtenidos con flagrantes violaciones de los derechos humanos y laborales tendían a ser, naturalmente, más bajos (algo que haría peligrar las empresas nacionales, obligadas legalmente a soportar

\footnotetext{
${ }^{12}$ Con efecto, cuando la cláusula social se incorpora en el ámbito del Sistema de Preferencias Generalizadas (Reglamento $n^{\circ}$ 978/2012 del Parlamento Europeo y del Consejo), no es necesario que el país en desarrollo conceda, en el mismo instrumento jurídico, reducciones arancelarias a los bienes exportados por la Unión Europea, ya que el acceso europeo a eses mercados se contempla en el marco de la cláusula de la nación más favorecida (NMF), principio básico de la Organización Mundial del Comercio heredado del GATT 1947. En el caso de los acuerdos comerciales recíprocos, ambas partes estarán sometidas a la cláusula NMF.

${ }^{13}$ Inclusive en la última etapa de la Ronda Uruguay del GATT, algo que, finalmente, no se logró en el ámbito multilateral.
}

estándares laborales más elevados y costosos, según Waer,1996) ${ }^{14}$.

Siempre ha existido por parte de los gobiernos, productores y trabajadores europeos el temor de que los productos importados de los países en desarrollo, con precios competitivos en virtud de los bajos salarios, pongan en peligro la supervivencia de las empresas y de los puestos de trabajo. Supuestamente la cláusula social minimizaría esta posibilidad en virtud de la obligación, asumida por el país en desarrollo signatario del acuerdo comercial con la Unión Europea, de respetar los derechos humanos y laborales de los trabajadores. Toda esta exaltación de los derechos humanos, incluyendo su potencial utilización como barrera comercial (la condicionalidad de la cláusula social) ${ }^{15}$ no es algo que parece haber surgido recientemente, como veremos en el epígrafe siguiente.

\section{LOS DERECHOS HUMANOS EN EL PROCESO HISTÓRICO}

La observación de la historia de las relaciones internacionales señala que los derechos humanos y su protección legal, en Europa y en América, están inmersos en procesos históricos que reflejan tanto la noción de "fuerzas

\footnotetext{
14 Waer, P. (1996). Social Clauses in International Trade-The Debate in the European Union. Journal of World Trade, 30(4), 25-42.

${ }^{15}$ Unión Europea en relación a América Latina y América Latina en relación a China.
} 
profundas de la historia" (Renouvin \& Duroselle, 2000) ${ }^{16}$ como el "estructuralismo temporal" (Braudel, 1986) ${ }^{17}$. Con efecto, existen poderosas fuerzas motrices de la historia que van arrastrando sedimentos diversos a lo largo de todo su recorrido de modo que la incorporación de la cláusula social en los acuerdos comerciales podría presentar tanto una vinculación histórica con el período de consolidación de los derechos humanos y laborales como con el posterior interés en proteger económicamente a los denominados "sectores sensibles" de la competencia "desleal" de los países cuyas empresas e industrias emplean mano de obra barata y/o en condiciones precarias ${ }^{18}$.

Ambas aportaciones están en la base del juego diplomático, político, económico y comercial y parecen señalar - especialmente en el caso de

\footnotetext{
${ }^{16}$ Renouvin, P. \& Duroselle, J. B. (2000). Introducción a la historia de las relaciones internacionales. México: Fondo de Cultura Económica.

${ }^{17}$ Braudel, F. (1986). La historia y las ciencias sociales. Madrid: Alianza.

${ }^{18}$ Además de la propia dinámica histórica, Duroselle (Duroselle, J. B., 1990, História da Europa. São Paulo: Círculo de Leitores) señalaba también que el papel de las circunstancias (eventos) en el proceso político decisorio debe ser considerado, algo que, en el caso tratado en este artículo, se puede asociar al hecho de que la Comunidad Económica Europea no vinculaba originariamente la protección de los derechos humanos a la cooperación en el caso de los países ACP, hasta que el general Idi Amin Dada procediera al genocidio de las minorías étnicas en Uganda o Jean Bedel Bokassa se involucrara con la matanza de los niños que protestaban contra la política del gobierno en Centroáfrica (régimen depuesto por iniciativa atribuida al gobierno galo de Valéry Giscard d'Estaing), de acuerdo con Kabunda (Kabunda, B. M., 2000, Derechos humanos en Africa: teorías y prácticas. Universidad de Deusto.
}

que la cláusula social distorsione el libre flujo internacional de comercio - que los intereses económicos llegan a distorsionar la compartición de valores denominados "universales". Esta compleja red de relaciones, según Saraiva $(2001)^{19}$, solamente puede ser comprendida adecuadamente desde la perspectiva histórica ${ }^{20}$. La historia de las relaciones internacionales, que permitiría comprender el proceso continuo que los derechos humanos y su protección desempeñan en el mundo actual, ha evolucionado mucho en las últimas décadas aunque la retomada de la globalización, con el fin del socialismo (y la bipolaridad de las relaciones internacionales), presenta escenarios muy dispares (Art \& Jervis, 1996) ${ }^{21} / /^{22} /{ }^{23}$. En su magistral obra

${ }^{19}$ Saraiva, J. F. S. (2001). História das relações internacionais: $O$ objeto de estudo e a evoluçāo do conhecimento. Relações internacionais — dois séculos de história. Brasília: IPRI, 15-58.

${ }^{20}$ Esto exige un enorme esfuerzo por parte del analista, puesto que la evolución de los procesos internacionales suele demostrar que la comparación, en términos metodológicos, no puede ser realizada fácilmente entre ambientes internacionales en que los derechos humanos aparecen como un elemento de importancia fundamental.

${ }^{21}$ Art, R. C. \& Jervis, R. (1996). International politics: enduring concepts and contemporary issues. New York: Harper Collins.

${ }^{22} \mathrm{Su}$ tradicional perspectiva, basada en la política y la diplomacia, ha sido reemplazada por un crisol que comprende la economía, el derecho, el comercio, la política, la cultura, la ecología, las identidades y los valores, entre otros factores.

$23 \mathrm{La}$ historia de las relaciones internacionales proporciona movimiento, sentido y racionalidad al presente y permite comprender la incorporación, consolidación y permanencia de ciertos valores en el ámbito europeo y mundial. Así, por ejemplo, retrotrayendo en el tiempo, observamos que la consagración de los derechos humanos plasmados durante la reconstrucción europea (en el período 
"Todo imperio perecerá: Teoría sobre las Relaciones Internacionales", Duroselle (1998) ${ }^{24}$ deja claro la necesidad del conocimiento histórico en el estudio de las relaciones internacionales contemporáneas (a propósito, Watson, $2009^{25}$, señalaba el concepto de sociedad internacional europea siglo XIX e inicio del siglo XX - para explicar la construcción de patrones de conducta en el juego de las relaciones internacionales a partir de la hegemonía colectiva ejercida por los estados europeos en las relaciones con terceros países en aquella época) ${ }^{26}$.

La noción de fuerzas profundas, desarrollada por Pierre Renouvin y publicada originalmente en 1953 (el primer volumen de los ocho que componen la obra) considera que la evolución de las relaciones internacionales corresponde a variados aspectos de la vida de la sociedad. Para Renouvin y algunos de sus colegas, como Gaston Zeller (1987) ${ }^{27}$ y André Fugier

inmediatamente posterior a la Segunda Guerra Mundial) se cristalizó en las relaciones interiores y en la política exterior del bloque hasta llegar a nuestros días, con la inclusión de la cláusula social en los acuerdos comerciales de la Unión Europea con América Latina.

${ }^{24}$ Duroselle, J. B. (1998). Todo imperio perecerá: Teoría sobre las relaciones internacionales. México: Fondo de Cultura Económica.

${ }^{25}$ Watson, A. (2009). The Evolution of International Society: A Comparative Historical Analysis. Londres: Routledge.

${ }^{26}$ Con características propias, la Unión Europea también tiene un patrón de conducta, consustanciado en una política exterior común y observable, en lo que nos interesa, en la inclusión de la cláusula social en los acuerdos comerciales.

${ }^{27}$ Zeller, G. (1987). Les institutions de la France au XVIe siècle (Vol. 14). Presses universitaires de France.
$(2007)^{28}$, coexisten diversos factores e intereses (políticos, económicos, comerciales, estratégicos y sociales) en ese ámbito que son tomados en cuenta por los hombres de estado en el cumplimiento de sus funciones. En este sentido, aunque originariamente sirviera para estimular la protección de los derechos humanos y laborales, la politización de la cláusula social la ha convertido en un instrumento jurídico capaz de jugar un rol económico importante a favor de la protección de los sectores sensibles de la Unión Europea (prueba de esto es que el Sistema de Preferencias Generalizadas atribuye reducciones arancelarias distintas a los bienes procedentes de los países en desarrollo en función de la sensibilidad de los sectores europeos). En otras palabras: la cláusula social funciona como una barrera comercial no arancelaria (en virtud de su condicionalidad) políticamente correcta al interferir en el libre acceso de bienes exportables por los países en desarrollo al mercado único europeo. Además, por las razones anteriormente expuestas, no parece tener mucha efectividad como elemento promotor de los derechos humanos y laborales en el interior de los mercados latinoamericanos (o de cualquier mercado de países en desarrollo) por la propia característica del liberalismo económico (de paso, fomentado

\footnotetext{
28 Fugier, A. (2007). La Guerra de las Naranjas: (Luciano Bonaparte en Badajoz). L. A. L. Píriz (Ed.). Diputación Provincial, Departamento de Publicaciones.
} 
tanto por la Unión Europea como por América Latina y estimulada y apoyada por organizaciones internacionales de vocación universal, como la Organización Mundial del Comercio).

La interrelación entre todos esos factores (políticos, económicos, comerciales, jurídicos, sociales, estratégicos) es esencial para comprender cómo se articula realmente la cláusula social en el comercio internacional. Se podría tomar por base la conocida crítica de Renouvin sobre la historia diplomática asentada en el hecho político y la biografía de grandes personajes, por considerarla insuficiente para explicar las catástrofes del siglo XX. Lo mismo pasa en relación a la cláusula social: está inmersa en un escenario que es un verdadero crisol de intereses. Analizar las relaciones internacionales, actualmente, implica analizar el complejo entramado de sus factores. No se puede olvidar que para Renouvin, uno de los más ilustres historiadores del siglo $\mathrm{XX}$, las fuentes de investigación necesitaban ser ampliadas para abarcar otros factores (como las fuerzas económicas) pues solamente así sería posible producir un conocimiento acorde con el dinamismo y la amplitud de la realidad internacional (su visión, innovadora en la época, plasma perfectamente la transversalidad de las relaciones internacionales). Por su vez,
Duroselle (1990) ${ }^{29}$, antiguo alumno de Renouvin, desarrolló la noción de "fuerzas profundas de la historia" (que incluía los factores económicos, entre otros) estableciendo su influencia sobre la formulación de las políticas exteriores. Según el autor, el factor económico ejercería su poder sobre los complejos escenarios en los cuales las grandes decisiones políticas son tomadas, característica cada vez más marcada en la sociedad internacional actual. Naturalmente, en relación a la cláusula social, los intereses económicos, comerciales, sociales etc. en juego son muy elevados, de modo que tanto su inclusión en un acuerdo comercial como el seguimiento y la interpretación de las medidas adoptadas por los países en desarrollo (ratificación y aplicación efectiva de los convenios de la ONU y la OIT sobre protección de los derechos humanos y laborales) han sido absorbidas por un proceso político que atiende a compromisos económicos y sociales históricamente consolidados (como la protección de los sectores sensibles de la economía, con destaque para la agricultura en el ámbito de la Unión Europea). Este seguimiento no puede ser jurídico por absoluta ausencia de competencia legal/jurisdiccional de la Unión Europea en apreciar debidamente temas jurídicos de terceros estados. Además, la presencia de abundantes instrumentos

\footnotetext{
29 Duroselle, J. B. (1990). História da Europa. São Paulo: Círculo de Leitores.
} 
normativos en el sistema interamericano de derechos humanos pone en entredicho, bajo la perspectiva jurídica, la presencia persistente de la cláusula social en los acuerdos comerciales concluidos con América Latina.

Históricamente, el liberalismo económico y la consolidación del bloque europeo como una formidable potencia comercial acabarían redefiniendo, según Paillard (1994) ${ }^{30}$, una posición de sostenimiento de las asimetrías entre los actores internacionales (países desarrollados y en desarrollo, base del conflicto norte-sur). De hecho, la cláusula social, aunque busque fomentar la ratificación de convenios de protección de los derechos humanos y laborales por los países en desarrollo, también contribuye al mantenimiento de un escenario asimétrico al funcionar como una barrera comercial no arancelaria capaz de interferir en el libre flujo y en el acceso de bienes originarios de América Latina (y de todos los países en desarrollo) en el mercado interior de la Unión Europea. Es interesante comprender como la cláusula social se articula en el comercio internacional para verificar las posibilidades y los límites que ofrece en términos de promoción de los derechos humanos y laborales en mercados caracterizados por el liberalismo económico.

\footnotetext{
${ }^{30}$ Paillard, Y. G. (1994). Expansion occidentale et dépendance mondiale: fin du XVIIIe - 1994. Paris: Armand Colin.
}

\section{CONCLUSIONES}

La eficiencia de la cláusula social en el mercado económicamente liberal es cuestionable, pues los agentes privados poseen capacidad decisoria en la asignación de recursos (lo que incluye los salarios) y preocupación en producir con bajos costes para enfrentar la competencia y conquistar clientes y cuotas de mercado. Por otro lado, la dinámica histórica señala la coexistencia de la protección tanto de los derechos humanos y laborales como de los intereses comerciales (con especial destaque para los sectores "sensibles", como la agricultura). En este sentido, la concesión de ventajas comerciales para el acceso de productos exportables por los países en desarrollo al mercado único de la Unión Europea (v.g., rebajas arancelarias), está directamente relacionada con la condicionalidad de respeto a los derechos humanos y laborales establecida por la cláusula social. Si por un lado esta cláusula cumple el rol de elemento promotor de los derechos humanos y laborales (cuestionable en la práctica) por otro, al establecer una condicionalidad, también interfiere en el libre flujo internacional de bienes y servicios, lo que la caracteriza como una barrera comercial no arancelaria. La conclusión a que se llega es que su presencia en los acuerdos comerciales Unión Europea-América Latina no es circunstancial, sino el resultado de un 
movimiento histórico políticamente

consistente.

\section{REFERENCIAS BIBLIOGRÁFICAS}

Art, R. C. \& Jervis, R. (1996).

International politics: enduring concepts and contemporary issues.

New York: Harper Collins.

Bahillo, C. G. (2012). Ética y responsabilidad empresarial en la sociedad globalizada. Revista Icade. Publicación de las Facultades de Derecho y Ciencias Económicas y Empresariales 75, 125-142.

Braudel, F. (1986). La historia y las ciencias sociales. Madrid: Alianza.

Chan, A. (1998). Labor standards and human rights: The case of Chinese workers under market socialism. Human Rights Quarterly, 20(4), 886904.

de la Garza, E. (2012). La

subcontratación y la acumulación de capital en el nivel global. pp. 17-40, en Celis J., (2012) Comp. La

Subcontratación Laboral en América Latina: Miradas Multidimensionales. Medellín, Colombia: Escuela Nacional Sindical.

de Rezende Martins, E. C. (2002). Relações internacionais: cultura e poder. Ibri.

Dunne, T., \& Wheeler, N. J. (1999). Human rights in global politics. Cambridge: Cambridge University Press.

Duroselle, J. B. (1990). História da Europa. São Paulo: Círculo de Leitores.
Duroselle, J. B. (1998). Todo imperio perecerá: Teoría sobre las relaciones internacionales. México: Fondo de Cultura Económica.

Fugier, A. (2007). La Guerra de las Naranjas: (Luciano Bonaparte en Badajoz). L. A. L. Píriz (Ed.). Diputación Provincial, Departamento de Publicaciones.

Ishay, M. R. (2008). The history of human rights: From ancient times to the globalization era. Univ of California Press.

Kabunda, B. M. (2000). Derechos humanos en Africa: teorías y prácticas. Universidad de Deusto.

Paillard, Y. G. (1994). Expansion occidentale et dépendance mondiale: fin du XVIIIe - 1994. Paris: Armand Colin.

Porter, M. (2008). Las cinco fuerzas competitivas que le dan forma a la estrategia. Harvard Business Review, 86(1), 58-77.

Renouvin, P. \& Duroselle, J. B. (2000). Introducción a la historia de las relaciones internacionales.

México: Fondo de Cultura Económica.

Saraiva, J. F. S. (2001). História das relações internacionais: $O$ objeto de estudo e a evolução do conhecimento. Relações internacionais - dois séculos de história. Brasília: IPRI, 1558.

Smith, K. E. (1998). The use of political conditionality in the EU's relations with third countries: How effective? European Foreign Affairs Review, 3(2), 253-274.

Waer, P. (1996). Social Clauses in International Trade - The Debate in 
the European Union. Journal of World

Trade, 30(4), 25-42.

Watson, A. (2009). The Evolution of

International Society: A Comparative

Historical Analysis. Londres:

Routledge.

Zeller, G. (1987). Les institutions de la France au XVIe siècle (Vol. 14).

Presses universitaires de France. 